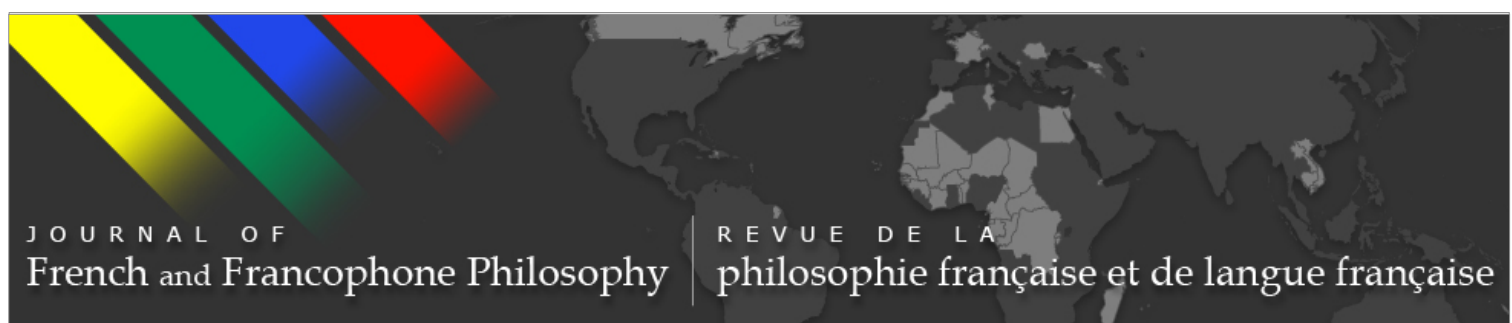

\title{
Après la chair
}

\section{Claude Romano}

Journal of French and Francophone Philosophy - Revue de la philosophie française et de langue française, Vol XXI, No 2 (2013) pp 1-29

\author{
Vol XXI, No 2 (2013) \\ ISSN 1936-6280 (print) \\ ISSN 2155-1162 (online) \\ DOI 10.5195/jffp.2013.611 \\ www.jffp.org
}

\section{(oc) EY-NG-ND}

This work is licensed under a Creative Commons Attribution-Noncommercial-No Derivative Works 3.0 United States License.

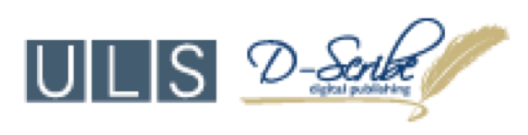

This journal is operated by the University Library System of the University of Pittsburgh as part of its D-Scribe Digital Publishing Program, and is co-sponsored by the University of Pittsburgh Press 


\title{
Après la chair
}

\author{
Claude Romano
}

Université Paris IV-Sorbonne

Sa chair spirituelle a le parfum des Anges.
- Baudelaire

Il n'y a pas de question plus urgente, pour la phénoménologie, que la question du "corps propre," comme il est convenu de l'appeler depuis Husserl. Mais il n'y a pas non plus de question qui ait été davantage négligée par les phénoménologues contemporains. À première vue, cette affirmation se heurte à l'évidence d'une production littéraire quasi exponentielle autour de cette notion depuis plus d'une trentaine d'années, aussi bien en histoire de la philosophie que dans des travaux qui se sont efforcés de croiser la perspective phénoménologique avec les apports des sciences du cerveau et de la cognition. L'ennui est que cette ample littérature ne pose aucune des questions préjudicielles à l'adoption du concept de corps propre ou de chair (Leib) en phénoménologie; pour l'essentiel, elle fait comme si ce concept allait de soi et se borne à se demander de quelle manière il pourrait "féconder" des approches scientifiques plus positives. La légitimité du concept même de Leib et de ses prolongements à l'intérieur du courant phénoménologique $\mathrm{n}^{\prime} \mathrm{y}$ est jamais questionnée en tant que telle. Non seulement on ne se demande pas si les descriptions de cette "chair" au fil conducteur de l'expérience du toucher redoublé est tenable, mais on ne soulève même pas la question de savoir si l'adoption de ce concept chez Husserl et ses successeurs n'est pas conditionnée par des présupposés discutables et, en vérité, par tout un cadre théorique, de sorte que la mise en question de certains aspects centraux de ce cadre devrait conduire inévitablement à une révision en profondeur de ce concept. C'est cette question que nous voudrions aborder dans ces pages. Compte-tenu de l'ampleur du sujet, notre but sera uniquement d'indiquer un certain nombre de directions que pourrait - ou devrait - emprunter la réflexion.

Journal of French and Francophone Philosophy | Revue de la philosophie française et de langue française Vol XXI, No 2 (2013) | www.jffp.org | DOI 10.5195/jffp.2013.611 


\section{Le cadre général: révolution scientifique et monde de la vie}

Pour apercevoir ce que la phénoménologie peut apporter de nouveau à une compréhension du corps, il faut commencer par remonter en deçà de la pensée de Husserl, à la révolution scientifique moderne qui, à maints égards, a fixé l'horizon à l'intérieur duquel la question du corps prend forme et reçoit son orientation fondamentale depuis le début des Temps modernes jusqu'à l'époque contemporaine. Ce qui caractérise en premier lieu cette révolution scientifique est l'adoption d'un concept de nature sans précédents dans l'histoire de la culture occidentale. Aux yeux de Galilée et de Newton, la nature n'est plus un domaine ontique particulier, celui des étants qui possèdent en eux-mêmes le principe de leur mouvement et de leur repos et qui, à cet égard, nous apparaissent comme "nature" du point de vue de notre intuition immédiate; c'est la totalité des systèmes matériels en mouvement relatif pour autant qu'ils se prêtent à une connaissance au moyen de l'observation, de la mesure et du calcul, c'est-à-dire pour autant qu'ils obéissent à des lois causales strictes, exprimables en langage mathématique. Cette nouvelle "nature" est une et immuable; elle embrasse au sein d'une même réalité homogène la chute d'une pierre et l'orbite de Jupiter et s'étend jusqu'aux confins de l'univers; elle ne laisse rien en dehors d'elle, pas même les artefacts, pas même les vivants et les hommes, qui deviennent, à leur tour, des "objets" soumis aux mêmes lois mathématisables. La révolution scientifique moderne entraîne ainsi dans son sillage tout un cortège de conséquences d'ordre philosophique. En dépouillant le cosmos de toute téléologie intrinsèque et en le réduisant à la totalité des systèmes matériels et à leurs interactions causales; en isolant des propriétés que l'univers possède indépendamment du sujet qui les connaît (les "qualités premières" de Locke), d'une part, et des propriétés qui ne lui échoient qu'en relation à notre esprit et à nos organes sensoriels, et qui ne sont, en réalité, que des déterminations de notre psychisme (les "qualités secondes"), de l'autre; en appelant "nature," en somme, tout ce qui est objectivement connaissable au moyen des équations de la science, la philosophie naturelle moderne déploie un nouveau concept d'objectivité qui expulse l'esprit humain de l'univers physique et ne laisse plus place qu'à deux possibilités: ou bien réduire cet esprit à la matière en concevant les phénomènes mentaux comme de purs effets de processus neurophysiologiques, selon la voie du matérialisme et du naturalisme; ou bien maintenir son hétérogénéité de principe en affranchissant l'esprit du mécanisme et du déterminisme naturels, selon le chemin emprunté par les dualismes et les idéalismes.

Dans cet univers neutre et indifférent, l'homme se sent abandonné, livré à un effroi et une angoisse auxquels la prose de Pascal a conféré leur expression définitive: "Le silence éternel de ces espaces infinis m'effraie." Seul en face du vide et de la matière infinie, il n'est plus qu'une réalité divisée et contradictoire, à la fois simple jouet du déterminisme universel

Journal of French and Francophone Philosophy | Revue de la philosophie française et de langue française

Vol XXI, No 2 (2013) | http://www.jffp.org | DOI 10.5195/jffp.2013.611 
par son corps, et lui résistant néanmoins par son esprit comme un îlot de liberté inexpugnable - mais inexplicable -, à l'image d'un Dieu transcendant. D'un côté, livré et assujetti sans reste aux lois de la mécanique, de l'autre, semblable à "une petite divinité dans son department," 3 selon la formule de Leibniz. Cette nouvelle image de la nature ressuscite dès lors certains motifs gnostiques. La gnose antique concevait l'esprit humain, le pneuma, comme une étincelle divine tombée du ciel et précipitée dans un corps périssable, comparé à un sépulcre, duquel seule une connaissance transcendante pouvait la libérer; elle concevait le monde comme un lieu d'aliénation, un bourbier ou un marécage, où rien ne rappelait plus le kosmos grec, avec ses connotations de parure, de beauté, d'ordre et de finalité; enfin, elle promettait un salut par le savoir, seul antidote à la puissance que la matière exerce sur l'esprit, puisque, avec l'abandon de la pronoia stoïcienne, ne subsistait plus qu'une loi cosmique aveugle et implacable: pour les gnostiques, comme le soulignera Plotin, les astres sont des tyrans. Or, la nouvelle gnose qui émerge de la révolution scientifique moderne place, elle aussi, l'esprit en dehors de la nature, réduite à la matière infinie, en le pensant ou bien comme son principe antagoniste (idéalisme, dualisme), ou bien comme le simple effet de processus causaux qui se déroulent en elle (matérialisme); cette nature est à nouveau conçue comme une réalité étrangère à l'homme, dépourvue de tout sens et de toute finalité, devant laquelle l'esprit ne peut que s'abîmer dans une perplexité et une angoisse sans bornes; enfin, nous retrouvons ici l'alliance caractéristique du savoir et de la puissance qui revêt à présent une signification nouvelle, bien résumée par la formule de Bacon selon laquelle "les deux fins jumelées, la Science et la Puissance humaines, aboutissent véritablement au meme," ${ }^{4}$ où le seul salut possible face à une nature qualifiée par Bacon d' "adversaire" n'est plus celui que procure une Révélation à l'usage de quelques initiés, mais celui que dispense le développement conjoint de la science et des techniques unies en un même culte prométhéen de l'ingenium humain. Le positivisme moderne avec ses relents mystico-religieux en constitue l'héritier direct.

On pourrait croire que cette nouvelle gnose se situe davantage du côté de l'idéalisme, avec sa tendance au solipsisme et le sentiment "sublime" qu'il procure d'un surplomb à l'égard du monde environnant, la solitude hautaine et glacée du sujet ayant ici pour contrepartie la puissance quasi démiurgique avec laquelle il "constitue" ce même monde, que du naturalisme ou des matérialismes; elle triomphe assurément dans les philosophies transcendantales. Mais, en réalité, la nouvelle gnose qui résulte de l'interprétation philosophique que la révolution scientifique moderne a donnée d'elle-même sur un de ses versants au moins, celui inauguré par Descartes et poursuivi par Kant et ses héritiers idéalistes, détermine tout le cadre à l'intérieur duquel prend place l'opposition entre monismes et dualismes, entre matérialisme naturaliste et idéalisme absolu ou transcendantal. Il ne faut pas s'y méprendre, en effet: le naturalisme et l'idéalisme partagent une même conception du monde (ou de la nature)

Journal of French and Francophone Philosophy | Revue de la philosophie française et de langue française Vol XXI, No 2 (2013) | http://www.jffp.org | DOI 10.5195/jffp.2013.611 
comme univers matériel soumis à des lois, d'où ont été exclus par principe tous les prédicats "humains," toutes les fins, significations et valeurs, tenues désormais pour des "projections" et des anthropomorphismes - la seule différence notable entre ces deux courants philosophiques étant que le naturalisme prétend dériver l'esprit humain lui-même, avec ses "qualités secondes" et les significations qu'il "projette" sur le monde, de l'univers physique réduit à ses "qualités premières," tandis que l'idéalisme soutient que cette dérivation est impossible. C'est le même concept de "monde" qui soustend les deux positions - un monde neutre et objectif dépouillé de tout ce qui le rend humain; sauf que ce monde est conçu tantôt comme la seule et unique réalité, et, partant, comme principe d'explication ultime des phénomènes de l'esprit, et tantôt comme laissant ces phénomènes, et l'esprit lui-même, en dehors de sa juridiction au titre d'une seconde réalité hétérogène et autosuffisante.

La proximité de ces deux positions se révèle en définitive dans le fait qu'elles soulèvent toutes deux le même problème: si la nature recèle seulement en apparence des significations et des fins qui ne sont, en réalité, que des "projections" anthropomorphiques; si les plantes semblent seulement rechercher la lumière, et les animaux paraissent mus par des tendances et des désirs qui confèrent aux objets de leur environnement telles ou telles significations, "à poursuivre" pour les uns et "à fuir" pour les autres, et si cette téléologie apparente est explicable sans reste par des principes mécaniques, ce n'est pas seulement la "nature" hors de nous, mais la "nature" en nous qui doit être tenue pour un anthropomorphisme. En d'autres termes, l'homme devient lui-même un anthropomorphisme, selon la juste formule de Robert Spaemann. ${ }^{5}$ L'homme est une illusion pour l'homme, et avec lui s'effondre le monde humain tout entier, le monde où prennent place les fins et les significations en général; seule demeure une nature absurde et étrangère. Mais, bien sûr, si l'homme est un anthropomorphisme, le concept d'anthropomorphisme est vidé de toute signification, puisque, avec la disparition de l'homme et son ravalement au rang d'illusion, il n'y a plus moyen de donner le moindre sens à l'idée d'une projection humaine de fins et de significations sur la nature infrahumaine. En somme, le naturalisme et l'idéalisme partagent une même idée, celle d'une possible suppression de tout prédicat humain qui laisserait subsister une nature intégralement objective, neutre à l'égard de toutes nos significations; mais cette idée, pour peu qu'on l'approfondisse, se révèle incohérente.

Les deux écoles aboutissent ainsi à une même impasse: celle qui consiste à tenir le monde phénoménal, le monde tel qu'il se présente à nous et que nous partageons au moins jusqu'à un certain point avec d'autres espèces, le milieu de notre vie, pour une pure et simple illusion, issue d'une projection illégitime sur une nature "en soi" de nos fins et de nos significations; mais, puisque ces fins et ces significations revêtent le statut d'illusions, au même titre que nous-mêmes, qui les projetons, il n'y a plus

Journal of French and Francophone Philosophy | Revue de la philosophie française et de langue française Vol XXI, No 2 (2013) | http://www.jffp.org | DOI 10.5195/jffp.2013.611 
moyen de comprendre comment cette projection elle-même opère; il est même difficile de savoir de qui ou de quoi cette illusion est l'illusion. Le monde phénoménal apparaît ainsi dépouillé de toute consistance propre, de toute vérité, y compris au niveau qui est le sien, celui d'un monde-pournous, c'est-à-dire du mode d'apparaître de la nature matérielle. Les significations qui peuplent notre monde et en font un monde humain doivent moins être expliquées que radiées de la réalité, sous le prétexte qu'il n'existerait qu'une seule description légitime de ce monde, sa description en termes physiques. Nous proposons d'appeler ce présupposé commun au naturalisme et à l'idéalisme sous ses différentes formes le "mythe de la description unique." Ce préjugé est un mythe dans la mesure où toute description, quels qu'en soient les objectifs et la portée, est dépendante d'intérêts, et les intérêts qui sous-tendent la description de l'univers en termes physiques ne sont pas moins prégnants que les autres. La description physique n'est qu'une description parmi d'autres du monde, certes revêtue d'un rôle central dans l'édifice de la science, mais qui, à l'instar de toute description, fait déjà intervenir nos fins, puisqu'elle envisage ce monde sous un rapport bien déterminé, tributaire d'intérêts bien déterminés, ceux qui ont trait à la nature de la matière et à ses lois. Même en décrivant l'univers en termes physiques, nous poursuivons des fins humaines particulières, et c'est ce qu'oublient aussi bien le naturalisme que l'idéalisme: le premier prétend partir de cette "description unique" pour en dériver l'esprit, le second s'oppose à cette dérivation tout en souscrivant aux prémisses qui la sous-tendent. C'est d'ailleurs la raison pour laquelle il lui est si difficile de triompher de son adversaire. La prémisse sous-jacente aux deux entreprises est en substance celle de tout positivisme: il serait possible de ramener la nature à de "purs faits" affranchis de toutes nos "valeurs." Ces faits ultimes seraient "la" réalité. Mais il n'y a rien de tel que "la" réalité, parce qu'il n'y a rien de tel qu'une description unique du monde; ce qui entraîne la ruine conjointe des deux positions. ${ }^{6}$

Ce que nous avons appelé "le mythe de la description unique" équivaut grosso modo à la position que Husserl a estampillée dans sa Krisis sous le nom d' "objectivisme." Cette tendance qui sous-tend non pas tant la révolution scientifique moderne que l'interprétation philosophique qu'celle-ci a donnée d'elle-même consiste à tenir les vérités de la physique pour l'expression d'un "en soi" des choses, et, par voie de conséquence, à considérer le monde phénoménal, le monde préscientifique dans lequel prend place notre vie, comme une pure et simple apparence. C'est ici que Husserl accomplit un véritable renversement de perspective. À travers son concept de Lebenswelt, il ne s'agit de rien de moins, en réalité, que de remettre en question de fond en comble, dans un geste philosophique d'une rare radicalité, tout le cadre conceptuel que nous avons tenté de reconstruire. Au lieu de postuler un "en soi" des choses, identifié à la nature physique, et de penser le monde phénoménal comme une simple apparence "subjective" issue d'une projection anthropomorphique, la dernière phénoménologie de Husserl

Journal of French and Francophone Philosophy | Revue de la philosophie française et de langue française Vol XXI, No 2 (2013) | http://www.jffp.org | DOI 10.5195/jffp.2013.611 
inverse l'ordre de priorité et proclame l'antériorité absolue du monde de la vie sur l'univers de la science et son autonomie à son égard. Non seulement la Lebenswelt possède sa propre consistance et sa propre vérité préscientifiques, mais elle est constamment présupposée par les théories scientifiques qui la prennent pour "sol" et point de départ. Or ce qui forme ainsi l'arrière-plan de la praxis scientifique et sur laquelle cette dernière ne cesse de faire fond ne peut se réduire à une simple chimère. Au lieu de scinder le monde en deux et d'y distinguer, ainsi que l'affirmait toute la tradition post-lockienne, des propriétés "objectives" et "subjectives," des "qualités premières" et des "qualités secondes," en tenant les unes pour vraies et les autres pour seulement apparentes, il faut affirmer au contraire que les "qualités premières" auxquelles s'intéresse la science sont le produit d'opérations méthodiques d'abstraction et d'idéalisation sur la base des soidisant "qualités secondes" et que, dans cette mesure, elles en présupposent la validité. Les couleurs phénoménales, par exemple, doivent posséder leur propre vérité au niveau de la perception naïve pour qu'il y ait un sens à leur faire correspondre un système de longueurs d'onde. Si les couleurs "n'étaient rien d'autre que..." (suivant la formule de tous les réductionnismes) des longueurs d'onde du spectre électromagnétique réfléchies par des surfaces, si le monde phénoménal devait être tenu pour "faux" au prétexte que l'univers physique est "véritable," loin d'être en présence d'un début d'explication physique des phénomènes perçus, nous aurions sapé le principe d'une telle explication. En substituant purement et simplement l'explanans à l'explanandum, cette pseudo-explication se détruit elle-même. Ainsi, au lieu d'opposer un monde vrai à un monde apparent, il convient de partir de la seule chose qui mérite d'être appelé un "monde," $c^{\prime}$ est-à-dire du milieu de notre vie, pour comprendre à partir de là en vertu de quelles opérations méthodiques d'abstraction et d'idéalisation la science peut jeter sur ce monde un "vêtement d'idées (Ideenkleid)" qui en exprime l'infrastructure mathématique. Les objets de la science continuent, en effet, de dépendre du monde de la vie; ils ne s'en affranchissent que tangentiellement. Le "mouvement" dont nous parlent les lois de Galilée, dépouillé par une suite de raisonnements a priori de tout ce qui pouvait encore le rattacher à notre monde de l'à-peu-près, et donc exempt de tout frottement et se déroulant dans le vide, continue malgré tout à renvoyer au mouvement tel que nous en faisons naïvement l'expérience, et il trouve dans ce renvoi son intelligibilité; les mesures "exactes" de la géométrie sont le fruit d'un passage à la limite à partir des mesures inexactes issues des techniques de l'arpentage, et ainsi de suite. Aux yeux de cette phénoménologie radicale, c'est donc l'univers physique qui doit être tenu pour dérivé et secondaire, pour une "construction" théorique — sans qu'il faille pour cela prendre position dans le débat opposant l'instrumentalisme, qui tient cette "construction" pour un simple outil de prédiction fiable, et le réalisme qui y voit la représentation fidèle d'une réalité inaccessible par d'autres voies.

Journal of French and Francophone Philosophy | Revue de la philosophie française et de langue française Vol XXI, No 2 (2013) | http://www.jffp.org | DOI 10.5195/jffp.2013.611 
Mais cette réévaluation sans précédent du monde phénoménal demeure, chez Husserl, une entreprise ambiguë. D'un côté, en affirmant que le monde de la vie avec ses significations humaines est le monde réel, elle constitue une remise en cause sans précédent du paradigme gnostique moderne issu de la révolution galiléenne. De l'autre, elle s'accomplit malgré tout à partir d'une position philosophique - celle de l'idéalisme transcendantal - qui est de part en part solidaire de ce cadre gnostique et en reconduit toutes les apories. Le monde de la vie tel que le conçoit Husserl n'est pas seulement le berceau des significations qui forment la toile de fond de nos vies et de nos pratiques; il est, plus profondément encore, ce qui est "constitué" par un ego transcendantal, lequel n'est ni dans le monde ni du monde mais en forme l'origine constituante. D'un côté, le monde de la vie échappe par sa consistance propre à toute projection anthropomorphique; de l'autre, les significations qu'il recèle lui sont malgré tout "conférées" par un sujet acosmique au moyen d'une donation de sens, d'une Sinngebung, sur la base de simples sensations, rebaptisées par Husserl "données hylétiques" ce pur "donné sensible" fournissant l'équivalent "psychique" des "faits bruts" postulés par le positivisme. La dualité épistémologique des données hylétiques et des actes conférant la signification est ici la conséquence directe de la reconduction du cadre scientifique moderne à l'intérieur d'une conceptualité destinée à interroger ce cadre dans son principe même. En réalité, l'idée de "sensation" entendue de cette manière est dépourvue de toute portée descriptive pour rendre compte du monde de la vie; ce n'est qu'un sous-produit de l'idéalisation scientifique, le corrélat psychique d'une stimulation tenue pour élémentaire afin de pouvoir être soumise à des protocoles expérimentaux reproductibles et contrôlables. Et, de fait, en soumettant le monde de la vie à sa problématique transcendantale d'une constitution de l'objectivité en général par la subjectivité, Husserl ne cesse d'injecter dans le monde de la vie sans s'en apercevoir des concepts issus de l'idéalisation scientifique. Cette ambiguïté fait du projet de la Krisis un projet constitutivement inachevé, qui ne pourrait être mené à son terme qu'à la condition de remettre en question tout l'arrière-plan transcendantal dont il procède.

Rien ne révèle mieux l'ambivalence de la position de Husserl que la tension symptomatique qui se fait jour entre deux de ses affirmations. D'une part, le monde de la vie est ce monde sans question qui se tient en deçà de toute théorie scientifique, et par conséquent aussi de toute "objectivité" au sens qui a été promu par la révolution galiléenne. Il contient des "éléments," l'air, le feu ou l'eau, des choses et des ustensiles, des hommes et des animaux, des étendues et des paysages, mais il ne contient, par principe, aucun "objet physique," puisque tout objet de ce type est engendré à partir des opérations abstractives de la science. De l'autre, pourtant, la problématique de la Lebenswelt est tout entière subordonnée à la problématique transcendantale de la "constitution," laquelle doit s'entendre comme constitution de l'objectivité en général, et l'objectivité ainsi conçue n'est

Journal of French and Francophone Philosophy | Revue de la philosophie française et de langue française Vol XXI, No 2 (2013) | http://www.jffp.org | DOI 10.5195/jffp.2013.611 
rien d'autre que l'objectivité propre aux sciences de la nature. En souscrivant à l'idéal cartésien d'une philosophie "scientifique" — idéal lui-même solidaire de la révolution scientifique moderne -, Husserl a cru nécessaire d'assujettir les phénomènes (et la phénoménologie tout entière) à l'idéal d'objectivité; il a décrété que la chose matérielle de la physique - et la nature elle-même en tant que domaine propre à l'investigation de cette science - devait être "constituée" comme une strate sui generis du monde apparaissant. Il a affirmé, en un mot, que "la "chose vraie" [...] est la chose de la physique, avec sa détermination logico-mathématique" ${ }^{7}$ et que cette "chose" affleure dès le niveau du monde perçu, dès notre inhérence au monde primordial: "même la transcendance supérieure de la chose physique n'implique point que l'on transgresse le monde tel qu'il est pour la conscience, ou pour tout moi [...] jouant le rôle de sujet de connaissance" ${ }^{\prime 8}$. Husserl tombe ainsi dans une contradiction insurmontable. Car l'idée de "monde de la vie" exigeait autre chose, elle exigeait que la transcendance au sens physique ne soit accessible que par le détour de l'objectivation, c'est-à-dire des opérations méthodiques abstractives qui sous-tendent la praxis scientifique, elle invitait à dire, bien plutôt, comme Husserl le reconnaîtra de plus en plus clairement, que les objets de la physique, les atomes, les électrons, les ions, etc., dont les seules déterminations sont de nature mathématique, reposent sur un passage à la limite grâce auquel nous quittons le "sol" du monde de la vie et lui substituons un royaume d'idéalités qui lui demeure à jamais hétérogène; bref, que les objets de la physique ressortissent à "un être transcendant à tout contenu de la chose tel qu'il s'offre à nous dans sa présence corporelle" ${ }^{\prime 9}$. D'un côté, donc, Husserl a conçu de plus en plus résolument l'objectivité comme le produit d'une idéalisation qui s'opère à partir du monde de la vie absolument antérieur et inobjectivable; de l'autre, il a appliqué à ce monde apparaissant lui-même des catégories issues de la pensée objectivante.

\section{Le corps phénoménal}

Cette difficulté a des conséquences directes — et profondes - sur ce que Husserl affirme du corps. On sait que, très tôt dans son parcours, le phénoménologue a distingué deux concepts de "corps" à la fois opposés et complémentaires: la chair ou corps propre (Leib) et le corps-objet (Körper). Le Körper est un simple objet matériel revêtu de déterminations physicobiologiques (extension, poids, solidité, organes remplissant des fonctions, etc.): il prend place tout entier dans la nature objective. La chair (Leib) est mon corps pour autant qu'il se révèle à moi dans une expérience singulière, celle du contact de ma main avec toute autre partie de mon corps, dans lequel la partie de ce corps auquel j'applique mon toucher (par exemple mon autre main) n'apparaît pas seulement comme "touchée," mais à son tour, comme siège de sensations tactiles, de sorte que nous avons ici affaire à un toucher redoublé dans chaque membre, où chacun d'eux apparaît alternativement touchant et touché, et où ces deux déterminations ne cessent de s'échanger et de se répondre en miroir. Tandis que le Körper appartient

Journal of French and Francophone Philosophy | Revue de la philosophie française et de langue française

Vol XXI, No 2 (2013) | http://www.jffp.org | DOI 10.5195/jffp.2013.611 
donc à une strate de l'expérience qui est celle de la "nature matérielle," la chair ressortit déjà à la nature animale, aux "aisthèta."

$\mathrm{Qu}^{\prime}$ en est-il du bien-fondé de cette distinction? Les remarques qui précèdent suggèrent déjà une difficulté fondamentale. Pour avoir une portée phénoménologique, une telle distinction suppose, en effet, que le corps-objet puisse être intuitivement donné dans notre monde primordial, "constitué" comme une strate de sa phénoménalité, autrement dit, qu'il apparaisse déjà au niveau du monde de la vie tel qu'il forme l'horizon de nos pratiques quotidiennes. Mais est-ce le cas? Si nous avons raison dans notre critique des présupposés résiduels issus de l'objectivisme qui continuent à sous-tendre la conception husserlienne de la Lebenswelt, la réponse doit être négative. Dans le monde de la vie conçu de manière radicale, il ne saurait y avoir d'objets physiques, et tout aussi peu des corps-objets. Des concepts physiques comme ceux de matière ou d'énergie, des concepts biologiques comme ceux de cellule ou de métabolisme ne trouvent aucune application dans ce monde naîf et procèdent uniquement de l'idéalisation scientifique. En somme, dans le monde de la vie, nous n'avons jamais affaire à des corps-objets en tant que substrats de déterminations physico-biologiques, mais toujours déjà à des corps propres en tant que modes d'auto-présentation de vivants de différentes sortes. ${ }^{10}$

Or, aux yeux de Husserl, la distinction chair/corps doit être pertinente au plan strictement phénoménologique d'une description du monde de la vie, et non pas au niveau des rapports complexes qui unissent - mais aussi séparent - cette description phénoménologique des "substructions" de la science. Nous devrions pouvoir être donnés à nousmêmes phénoménologiquement en tant que corps-objets dans le monde de la vie tout comme, selon les Ideen...I, nous devrions pouvoir "constituer" perceptivement la chose de la physique elle-même. Mais si la seconde affirmation est fausse, la première l'est aussi: il n'y a qu'une chose qui relève de plein droit du monde de la vie, c'est notre corps "propre" ou "phénoménal." Ce qui nous trompe là-dessus est que les objets de la biologie, de l'anatomie ou de la physiologie se définissent encore malgré tout par leur référence au monde perçu et au langage quotidien qui permet de l'articuler. En un sens, les “organes" de l'anatomiste sont aussi ceux que nous pouvons découvrir si nous pratiquons une dissection, les cellules du biologiste, celles que nous pouvons observer au microscope, et ainsi de suite. En d'autres termes, il n'y a pas de distinction tranchée entre les choses qui peuplent notre monde quotidien et les objets de la science, ni entre les jeux de langage de nos pratiques spontanées et ceux qui président au discours scientifique. Cette continuité entre l'expérience naïve et la théorie, entre le langage préscientifique qui permet d'articuler la première et le langage scientifique, ne doit pas pour autant nous faire croire que des concepts comme ceux de "cellule" ou d'"organisme" auraient déjà leur place au niveau de la Lebenswelt elle-même. L'antériorité de principe du monde de la vie et son autonomie par rapport aux idéalisations de la science équivaut en

Journal of French and Francophone Philosophy | Revue de la philosophie française et de langue française Vol XXI, No 2 (2013) | http://www.jffp.org | DOI 10.5195/jffp.2013.611 
réalité à l'asymétrie qui existe entre les descriptions scientifiques et les descriptions ordinaires du monde - asymétrie en vertu de laquelle les descriptions scientifiques reposent sur les descriptions ordinaires et les présupposent, mais la réciproque n'est pas vraie. Ainsi, nous pouvons parfaitement habiter notre monde et nous y orienter, identifier les choses qui nous entourent et apprendre à les reconnaître sans posséder le concept de "cellule" ou d'"organisme" (beaucoup de peuples ont été dans ce cas), tandis que nous ne pouvons pas posséder le concept de "cellule" ni identifier ces objets au microscope si nous ne pouvons pas identifier les choses de notre environnement vécu. Les objets de la biologie ne sont d'ailleurs pas situés sur le même plan que les choses de notre monde préthéorique: ce sont des objets dépendants d'hypothèses et "produits" par elles. Quelle que soit l'abondance de preuves dont nous disposions en faveur de la théorie cellulaire de l'organisme, il n'en reste pas moins que des hypothèses explicatives interviennent ici dans l'identification de ce que nous avons sous les yeux (ou sous la lentille de notre microscope); mais aucune hypothèse n'intervient quand nous identifions perceptivement un corps vivant dans notre environnement perceptif. Les choses de notre Lebenswelt possèdent une "certitude" radicale qui précède tous les justifications que nous pouvons donner et, a fortiori, toutes les hypothèses que nous pourrions forger à leur sujet, et qui pourraient se révéler vraies ou fausses. Leur certitude sans question se tient donc en deçà de toute théorie quelle qu'elle soit.

Mais si le Körper n'est rien d'autre qu'un sous-produit des opérations méthodiques d'abstraction et d'idéalisation propres aux sciences de la nature, il en résulte deux conséquences majeures. Tout d'abord, une conséquence interne à la phénoménologie: il n'y a plus de place pour la question de la "constitution" du Körper à même le monde de la vie comme une "strate" de sa phénoménalité, distincte de celle du Leib et dépendante de cette dernière, ni par conséquent pour le problème de 1"'objectivation" du Leib en tant que tel. Ensuite, une conséquence relative au potentiel critique de l'approche phénoménologique par rapport aux formulations traditionnelles du problème des "relations de l'âme et du corps," comme on a coutume de le désigner. Ces formulations partent du corps physique et physiologique comme de l'unique réalité pour se demander ensuite s'il est le siège de la conscience ou si la conscience lui demeure hétérogène. Or, c'est justement ce présupposé que la phénoménologie remet en question. Loin que notre corps-sujet tel que nous l'appréhendons du point de vue de notre expérience primordiale ne soit qu'une apparence subjective, à la différence du corps "véritable" de l'anatomie et de la physiologie, il lui est rigoureusement préordonné: le corps physiologique et tous les concepts permettant de le décrire ne font sens qu'en référence à un corps originairement expérimenté sur un plan préscientifique et auquel renvoient aussi nos "jeux de langage" ordinaires. Ce corps possède une "certitude" antérieure à toute croyance que nous aurions à fonder ou à justifier et à toute

Journal of French and Francophone Philosophy | Revue de la philosophie française et de langue française Vol XXI, No 2 (2013) | http://www.jffp.org | DOI 10.5195/jffp.2013.611 
hypothèse. Nous devons apprendre à le décrire pour lui-même, si nous voulons comprendre le corps-objet auquel se réfère la science.

Mais comment décrire ce corps? Appelons-le désormais "corps phénoménal" pour éviter toute équivoque avec la conceptualité de Husserl. Un premier trait de sa phénoménalité, qui concerne aussi bien mon propre corps que tout autre corps vivant, pourrait être le suivant: dans le monde quotidien et préscientifique, le corps n'apparaît pas comme une entité qui prendrait place aux côtés d'une autre entité qu'il faudrait appeler "âme" ou "esprit," il est le mode selon lequel un vivant se manifeste, il est la manière même dont s'accomplit toute autoprésentation d'un vivant qui apparaît, sous ce rapport, identique à son corps. Bien sûr, rien ne nous oblige à décrire un homme sous son aspect corporel; nous pouvons nous intéresser à lui sous d'autres rapports, celui de ses intentions et de ses buts, par exemple, et nous en donnerons alors une description en termes psychologiques; toutefois, si nous l'envisageons sous le rapport de son corps, son corps n'apparaît pas comme une partie de cet être, ni comme une possession qui lui échoit, il constitue la totalité de ce vivant. Le corps est par conséquent à la fois un "aspect" du vivant et quelque chose d'identique avec ce vivant considéré en totalité. Par exemple, c'est à l'être humain comme un "tout," c'est-à-dire à un corps pourvu de certaines capacités, que nous attribuons des aptitudes intellectuelles, de sorte que la question d'une "division" ou d'une "union" entre l'esprit et le corps ne se pose même pas à ce niveau; elle ne se pose que si nous réifions cet esprit et cette conscience et les envisageons comme des entités à part.

Cependant, entre l'expérience de mon corps phénoménal et celle des autres corps vivants se manifeste une différence essentielle. Mon corps, en effet, est éprouvé et ressenti d'une manière qui diffère entièrement de celle des autres corps. La phénoménologie husserlienne a situé cette différence dans le phénomène de l'auto-contact et dans cette particularité de l'expérience tactile de mon propre corps que Merleau-Ponty a baptisée "la réversibilité du sentir." Toutefois, il n'est pas évident que cette expérience soit décrite d'une manière satisfaisante par la phénoménologie husserlienne et post-husserlienne, ni d'ailleurs qu'elle soit aussi fondamentale qu'on ne l'a cru pour la caractérisation du corps phénoménal. Plusieurs points de cette description font manifestement problème: 1) le primat qu' elle confère à un sens, le sens tactile; 2) le concept de "sensation" auquel elle a recours, par exemple lorsqu'elle définit la chair comme le "soubassement hylétique (hyletische Unterlage)" de la conscience ${ }^{11}$; 3 ) la caractérisation de la chair comme un objet, certes un objet particulièrement "intime," ayant un statut tout à fait particulier, puisqu'il n'est pas donné de manière inadéquate à travers un flux d'esquisses, à la différence des objets transcendants - mais un objet malgré tout, "l'objet subjectif (das subjektive Objekt)," comme diront les Ideen... III, "par rapport auquel tous les autres objets (Objekte) sont des vis-à-vis, sont des objets environnants ("gegenüber" umgebende Objekte sind)."12 Comme nous allons le voir, ces trois caractéristiques de la chair 
husserlienne soulèvent de graves problèmes et, en dernière instance, doivent être rejetées.

Plutôt que de les examiner séparément, il est toutefois préférable de tenter de ressaisir le mouvement vif de toute cette description pour y discerner le rôle tenu par chacune d'elles. En effet, si l'on repart du texte "canonique" des Ideen... II, il est frappant que le point de départ de toute la description soit une conscience intentionnelle dont rien n'indique encore qu'elle soit liée à un corps ni "logée" en lui. Cette conscience est peuplée de sensations ou de "matériaux hylétiques" issus des différents sens. Toute la question est alors de savoir, parmi ces sensations, lesquelles doivent être rapportées à nous-même, c'est-à-dire localisées dans notre chair, et lesquelles se rapportent au contraire aux objets environnants. C'est au sens tactile qu'est dévolue la tâche de rendre compte de cette différence. En effet, ma main est considérée au début de l'analyse comme un objet parmi d'autres, une simple chose physique; mais, lorsque j'en viens à toucher cette main avec mon autre main, se produit un phénomène remarquable: cette main est "reconnue" comme mienne en vertu de la localisation en elle de sensations tactiles. C'est mon autre main qui, en l'effleurant, éveille en elle, pour ainsi dire, une sensibilité en sommeil qui répond à son mouvement d'exploration et fait que cet organe devient chair:

Mais en touchant ma main gauche, je trouve aussi en elle des séries de sensations du toucher, elles sont "localisées" en elle mais ne constituent pas des propriétés (comme le sont le rugueux ou le lisse de la main en tant que chose physique) [...] Si je les y ajoute, il n'est alors nullement question de dire que la chose physique s'enrichit, mais bien qu'elle devient chair (es wird Leib), qu'elle sent (es empfindet). ${ }^{13}$

Plusieurs points méritent d'être relevés. D'abord, c'est le toucher et lui seul qui est investi de la fonction de nous faire découvrir notre corps (Körper) comme "nôtre," c'est-à-dire comme chair (Leib). Ensuite, le toucher ne peut aboutir à ce résultat que parce que des sensations, ou, selon la terminologie de Husserl, des data hylétiques tactiles, se localisent dans ma main passive sous la caresse active de mon autre main. Il n'y a d'appréhension (ou de "constitution") de mon corps comme chair qu'au niveau hylétique: la chair est le "soubassement hylétique" de la conscience. Enfin, et c'est sans doute le plus remarquable, toute la description procède comme si ma chair, au départ, était un simple corps parmi les corps, c'est-à-dire n'était pas du tout ma chair; car mon corps "lui aussi est perçu de l'extérieur, écrit Husserl, quoique dans certaines limites." ${ }^{\prime 14}$ On dira sans doute qu'opère ici une abstraction méthodique de tous les prédicats proprement charnels visant à faire apercevoir la dynamique de leur constitution et préparant ainsi le "coup de théâtre" final: d'objet qu'elle était, ma main "devient chair," comme la Belle au bois dormant s'éveille d'un long sommeil sous le baiser

Journal of French and Francophone Philosophy | Revue de la philosophie française et de langue française

Vol XXI, No 2 (2013) | http://www.jffp.org | DOI 10.5195/jffp.2013.611 
du prince. Mais même si l'on accepte cette lecture, la possibilité d'une telle "abstraction" est hautement révélatrice du contexte transcendantal où prend place toute la description. Après tout, mon corps ne peut être appréhendé ainsi "de l'extérieur," pour reprendre la formule de Husserl, que parce qu'il est d'une certaine manière extérieur à moi-même. En régime transcendantal, en effet, j'ai un corps, mais je ne suis pas mon corps: la chair n'est pas une “composante du moi (Bestandstück des Ich),"15 elle demeure "étrangère à l'ego pur (Ichfremde)."16 En somme, ma chair est quelque chose que je "possède," une dépendance de mon ego pur, mais elle " $n$ 'appartient pas au domaine de l'égoïque (Ichliche) proprement dit"; elle n'est que "le tout premier "avoir subjectif" de l'ego,"17 lequel demeure la seule subjectivité au sens authentique et originaire. Réfractaire à toute inclusion dans le monde, l'ego pur ne s'incarne qu'à titre secondaire dans une chair et lui demeure toujours par principe irréductible. Mais ce n'est pas tout. Mon propre corps ne m'apparaît pas seulement dans une quasi extériorité, tout au long de l'analyse, il est caractérisé en outre comme une chose physique. On retrouvera ce même présupposé dans une description qui, à l'instar de celle de Merleau-Ponty, refuse le point de départ d'un pur ego transcendantal: "Quand ma main droite touche ma main gauche, je la sens comme une “chose physique," écrit Merleau-Ponty, mais au même moment, si je veux, un événement extraordinaire se produit: voici que ma main gauche aussi se met à sentir ma main droite, es wird Leib, es empfindet." 18

À ces affirmations, on pourrait opposer trois thèses: 1) l'abstraction d'un corps physique qui "devient chair" du fait de la localisation en lui de sensations du toucher est une abstraction illégitime, parce qu'il n'y a aucune place au niveau du monde de la vie pour un corps physique en général, ni a fortiori, pour un corps physique qui serait "le mien" (à supposer que cette dernière affirmation soit intelligible); 2) le primat conféré dans cette description au toucher est, lui aussi, discutable; il découle en grande partie d'un autre présupposé sous-jacent à la description, celui d'après lequel il faudrait constituer notre corps comme un "objet" sui generis, et donc lui appliquer de l'extérieur une fonction de connaissance. À cela, il faut répondre que notre corps est expérimenté comme "nôtre" dès le départ sans le détour d'aucune objectivation, et au moyen d'une expérience dont la particularité est qu'elle est précisément sans contrepartie objective, c'est-à-dire n'est en aucun cas une expérience d'objet; 3) enfin, pour décrire comment s'accomplit cette expérience non pas tant de notre corps, que de nous-même en tant que corps, il faut pouvoir distinguer deux régimes de sensibilité.

Si mon corps se révèle à moi d'entrée de jeu comme la localité primordiale que j'occupe, c'est d'abord en vertu d'une différence radicale entre la sensibilité par laquelle je me rapporte à ce corps et ma sensibilité générale au monde qui m'environne - une différence qui n'a guère été relevée en phénoménologie à notre connaissance. On peut appeler la première "sensibilité autocentrée" et la seconde "allocentrée." Par exemple, il est tout à fait différent d'avoir froid et de ressentir le froid d'un objet au 
contact de notre peau. Lors d'une forte chaleur, cette "sensation de froid" que procure une poche de glace peut être plaisante, alors que le fait d'avoir froid est toujours désagréable. La sensation d'avoir froid n'est pas la sensation du froid comme qualité d'un objet, c'est une sensation sui generis. On pourrait parler ici également d'une sensibilité "affective," dans la mesure où ce qui est ressenti est nécessairement connoté comme "agréable" ou "désagréable," ce qui n'est pas le cas de toutes les qualités perçues du monde. Pour prendre d'autres exemples, il y a une différence très nette entre la dureté d'un objet et la sensation de pression que nous éprouvons à son contact et qui peut aller jusqu'à une douleur si la pression continue à augmenter, ou encore entre l'effleurement par un corps étranger et l'impression de chatouillement qui en résulte. Ou encore, il existe une différence typique entre la forte chaleur d'un corps et la sensation de brûlure qu'il occasionne, entre l'intensité de la lumière ambiante et la sensation d'éblouissement qu'elle procure, et ainsi de suite. Cette différence entre des perceptions et des sensations affectives correspondantes est bien marquée par le langage, qui possède généralement des termes distincts pour les nommer. L'éblouissement n'est pas seulement la perception d'une lumière vive, mais une espèce de douleur oculaire ou de brûlure qui en résulte - mais pas dans tous les cas. Le chatouillement est autre chose que la perception de l'effleurement, et le fait d'avoir (trop) chaud est irréductible à la chaleur comme qualité atmosphérique.

Or, cette différence entre une sensibilité "affective" et autocentrée et une sensibilité "cognitive" et allocentrée est essentielle pour appréhender le corps phénoménal en tant que distinct des corps environnants. Mon corps phénoménal, en effet, se révèle en premier lieu comme la localité primordiale où se localisent des sensations affectives particulières: sentiment d'avoir chaud et froid, douleurs, plaisirs d'un certain type, chatouillements, démangeaisons, brûlures, faim et soif, mais aussi "sensations kinesthésiques," sensation de ma posture et de la position de mes membres, sensation de pression, de contraction musculaire, de faiblesse, de fatigue, d'essoufflement, de fourmillements dans un membre, voire sensation des manifestations symptomatiques des émotions et des affections en général: palpitations, sentiment d'oppression, d'atonie, de paralysie, excitation sexuelle, etc. Dans la mesure où cette sensibilité affective diffère d'entrée de jeu de la sensibilité "cognitive" vouée au monde et à la saisie de ses caractéristiques, et dans la mesure où ce régime de la sensibilité ne porte que sur mon corps comme la localité primordiale que j'occupe, ce corps étant le seul "lieu" où de telles sensations peuvent voir le jour, mon corps m'apparaît d'entrée de jeu comme absolument distinct de tout le reste, il n'y a et il ne peut y avoir entre lui et d'autres corps aucune confusion possible. Mon corps n'est pas d'abord un objet (un Körper) qu'il me faudrait ensuite $\mathrm{m}^{\prime}$ approprier et $\mathrm{m}^{\prime}$ attribuer sur la base d'une expérience tactile sui generis, il est le site où se localisent un certain nombre de sensations différant toto caelo de toutes les perceptions du monde - sensations qui ne trouvent leur place

Journal of French and Francophone Philosophy | Revue de la philosophie française et de langue française Vol XXI, No 2 (2013) | http://www.jffp.org | DOI 10.5195/jffp.2013.611 
qu'en lui. Il n'y a pas d'abord des sensations en général dont il faudrait ensuite pouvoir expliquer par quel mystère elles se rapportent à moi-même et à mon propre corps; la sensibilité affective est au contraire d'entrée de jeu la manière même dont j'éprouve mon corps comme tel ou, mieux, dont je m'éprouve moi-même corporellement, par exemple dont je m’éprouve corporellement affecté et blessé par la lumière (éblouissement) ou agréablement sollicité par la caresse du vent (chatouillement). C'est parce que mon corps m'est donné d'entrée de jeu dans le registre de cette sensibilité affective qu'il est absolument distinct de tout ce qui n'est pas lui et qu'il n'est justement pas un "objet" qu'on pourrait m'attribuer, un objet particulièrement intime (un "objet subjectif," selon la formule de Husserl), mais le volume que j'occupe et que je suis seul à pouvoir occuper, le lieu même de mon exposition aux autres et au monde. Il est l'ici de ma présence aux choses; il est ma "place au soleil," si l'on peut dire.

Nous comprenons mieux, désormais, pourquoi l'expérience du toucher redoublé est très largement insuffisante pour rendre compte de notre incarnation dans le monde. En premier lieu, cette "réversibilité du sentir" n'est qu'un cas particulier de la sensibilité affective au sens que nous avons conféré à ce terme. Lorsque ma main effleure mon autre main, la sensation de palpation relève du toucher actif comme modalité sensorielle cognitive ou gnosique, allocentrée, et il en va de même de la rugosité de ma main en tant que qualité perçue; en revanche, la sensation de pression éprouvée dans ma main passive, la sensation d'"être touché," si l'on veut, relève de cette modalité de ma sensibilité affective autocentrée qui me permet de m'éprouver moi-même continument et en toutes circonstances y compris lorsque je ne m'applique à moi-même aucun toucher actif -, par exemple en ressentant le contact d'un vêtement contre ma peau ou, lorsque je nage, l'enveloppement par l'élément liquide. Ainsi, je n'ai nul besoin d'une exploration tactile de mon corps pour pouvoir m'éprouver en tant que corps phénoménal. Le primat d'un toucher actif qui fait dire à Husserl que "la chair (Leib) ne peut se constituer en tant que telle originairement que dans le toucher et dans ce qui trouve sa localisation avec les sensations du

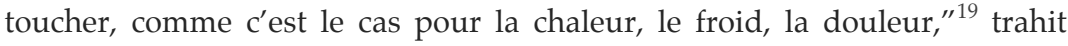
plutôt, dans sa description, le primat d'une connaissance de soi "en troisième personne" qui devrait passer par une forme d'objectivation de soi, c'est-à-dire le présupposé selon lequel je devrais m'appliquer à moi-même, pour ainsi dire "de l'extérieur," une fonction de connaissance du même type que celle que j'applique à des objets pour pouvoir m'éprouver moi-même et éprouver ma propre incarnation. Ma connaissance de mon corps devrait pouvoir être pensée sur le même modèle que celle que je prends de n'importe quel objet du monde, sauf que, dans ce cas particulier, l'objet connu serait un objet particulièrement "intime," un "avoir" primordial de l'ego pur.

Certes, la possibilité d'un "toucher double" au sens de Husserl existe indiscutablement, mais elle relève d'une attitude assez artificielle; elle n'est

Journal of French and Francophone Philosophy | Revue de la philosophie française et de langue française Vol XXI, No 2 (2013) | http://www.jffp.org | DOI 10.5195/jffp.2013.611 
qu'une manière particulière, et au demeurant assez rare, dont j'éprouve ma propre corporéité. Le plus souvent, cette corporéité se dévoile plutôt à moi dans une expérience dont le trait le plus saillant est qu'elle est au contraire dépourvue de tout corrélat objectif, c'est-à-dire telle qu'il n'y a ici aucun écart ou distance entre moi et moi-même, puisque, en ressentant une douleur dans mon épaule, par exemple, je ressens par là même mon épaule comme douloureuse, et en ressentant dans la poitrine l'oppression caractéristique de l'anxiété, je m'éprouve moi-même comme tendu et anxieux. Je n'ai d'ailleurs à accomplir aucune "localisation" de quoi que ce soit - y compris de la moindre sensation. La sensation douloureuse désigne son propre lieu, elle est localisée d'entrée de jeu, même si cette localisation est parfois vague et imprécise (on dira que la douleur "irradie"), et je n'ai nul besoin de rechercher son emplacement au moyen du sens tactile. Littéralement, il est faux de dire que "la chair [...] ne devient chair que parce qu'il y a, par le toucher, insertion des sensations, parce qu'il y a insertion des sensations de douleur, etc., en bref par la localisation des sensations en tant que sensations," ${ }^{20}$ car cette "insertion" et cette "localisation" n'ont jamais lieu. Plus généralement, il n'y a pas à "constituer" notre corps phénoménal comme un objet particulier; nous l'éprouvons directement comme nôtre ou l'éprouvons comme nous - sans le détour d'aucune objectivation, et il nous apparaît, de ce fait même, comme impossible à confondre avec aucune autre chose, comme le lieu même de notre présence dans le monde et au monde.

Mais, demandera-t-on peut-être, n'avons-nous pas nous-même succombé à un danger que nous avons plusieurs fois dénoncé, celui consistant à introduire dans le monde de la vie des concepts issus de la pensée objectivante, et en l'occurrence le concept de sensation? Nullement, en vérité. Car la sensation dont nous parlons n'a rigoureusement rien à voir avec celle que Husserl postulait à la base de toute "constitution." Le concept de sensation peut revêtir en effet deux significations bien distinctes. Selon le premier sens, la sensation n'est rien d'autre que le corrélat psychique d'une stimulation elle-même conçue comme atomique. On postule alors que notre perception tout entière se compose de sensations. On introduit dans le concept phénoménologique de "perception" l'abstraction forgée par la psychologie empirique pour tester ses hypothèses - par exemple, des hypothèses relatives à la perception de la couleur en vision monoculaire. L'ennui est que, du point de vue phénoménologique, des "sensations" conçues de cette manière ne se découvrent nulle part, ou presque; la perception a affaire dès le départ à des choses et à des caractéristiques-de-choses pourvues pour nous de significations vitales. En postulant des "data hylétiques" à la base de toute perception, Husserl a fondé toute sa description de la "constitution" du monde objectif sur un postulat issu de l'objectivisme. Mais il y a un autre usage que l'on peut faire du mot "sensation," beaucoup plus proche de son usage courant et préphilosophique. Une douleur, une brûlure, une démangeaison sont des

Journal of French and Francophone Philosophy | Revue de la philosophie française et de langue française Vol XXI, No 2 (2013) | http://www.jffp.org | DOI 10.5195/jffp.2013.611 
"sensations" dans la mesure où elles exemplifient trois caractéristiques: 1) elles sont ressenties en un lieu du corps phénoménal; 2) elles ne sont susceptibles de se produire que dans ce corps à l'exclusion de tout autre lieu; 3) elles possèdent effectivement une dimension "atomique," à la différence de notre perception du monde qui est un phénomène holistique: on parlera volontiers d'une sensation "isolée." Ces trois déterminations permettent de circonscrire un sens strictement phénoménologique du mot "sensation" qui ne succombe à aucun des dangers précédemment mentionnés et dont aucune description ne peut faire l'économie. S'il fallait décrire plus avant cette sensibilité autocentrée sous-jacente à notre expérience corporelle de nous-mêmes, il faudrait distinguer en elle plusieurs registres: les uns pour lesquels cette sensibilité revêt une forme plus holistique, comme dans le sentiment global de notre posture (ce que la psychologie du début du XXe siècle appelait notre "schéma corporel"), les autres pour lesquels cette sensibilité se monnaie en "sensations" relativement isolées et indépendantes les unes des autres (douleurs, démangeaisons, brûlures, etc.).

Ainsi, à travers cette sensibilité affective toujours connotée positivement ou négativement (suivant les modalités de l'agréable et du désagréable, du plaisant et du douloureux, du bien-être et du mal-être), se dessine une forme d'intériorité ou d'intimité de notre corps phénoménal. Mais cette "intériorité" n'a pas grand chose à voir avec celle qui est traditionnellement dévolue à la conscience. Mon corps occupe un volume spatial qui prend place à l'intérieur du monde, aux côtés des autres choses, il n'est pas situé sur un théâtre intérieur. Et l'"intimité" de mon corps est pour moi une caractéristique déjà spatiale, déjà mondaine, une manière dont je prends place à l'intérieur d'un environnement perçu, puisque aussi bien mon corps s'arrête là où commencent les choses. Il est vrai que les limites de mon corps sont poreuses. Mon corps s'annexe par exemple des instruments qui en forment le prolongement immédiat. La canne de l'aveugle, le sabre manié dans certains arts martiaux deviennent un appendice à nos pouvoirs corporels, une antenne au bout de laquelle le monde s'annonce, des organes surajoutés pourvus d'une véritable puissance tactile. Cependant, les limites de mon corps ont beau être poreuses, elles n'en existent pas moins, ce qui place mon corps dans le monde, à côté des autres étants, et non sur l'autre rive d'un Léthé qui me séparerait d'eux à jamais. L'intimité de mon corps est en quelque façon une dimension du monde lui-même. Elle n'a rien à voir avec la soi-disant "clôture égologique" d'une conscience transcendantale. Or, ce volume que j'occupe avec mon corps possède une caractéristique remarquable qui demande à être précisée: il est originairement structuré par des différences spatiales. La fameuse latéralisation droite/gauche qui a fait le désespoir de Kant, les différences entre "haut" et "bas," "devant" et "derrière" trouvent leur enracinement dans la spatialité primordiale de mon corps et en dépendent dans leur principe. Comme nous l'avons vu, mon corps phénoménal est structuré spatialement de telle manière que les différentes sensations affectives s'ordonnent spontanément en lui, qu'elles 
s'y localisent, si l'on peut dire, sans nul besoin d'y être localisées. Ma douleur m'apparaît d'entrée de jeu située dans ma main ou mon épaule; plus généralement, l'espace de mon corps est tel que je m'y retrouve d'emblée sans avoir besoin de m'y orienter. Et il en va de même des "ustensiles" que s'annexe mon corps: mon chapeau est situé sur ma tête, ma canne au bout de mon bras, sans que j'aie besoin, à aucun moment, de les situer par rapport à mon corps ou les uns par rapport aux autres. Comme l'écrit justement Merleau-Ponty, "si je suis debout et que je tienne ma pipe dans ma main fermée, la position de ma main n'est pas déterminée discursivement par l'angle qu'elle fait avec mon avant-bras, mon avant-bras avec mon bras, mon bras avec mon tronc, mon tronc enfin avec le sol. Je sais où est ma pipe d'un savoir absolu, et par là je sais où est ma main et où est mon corps, comme le primitif dans le désert est à chaque instant orienté d'emblée sans avoir à se rappeler et à additionner les distances parcourues et les angles de dérive depuis le depart." ${ }^{21}$ Parler d'un savoir "absolu" est cependant problématique. Il peut arriver, en effet, que je me trompe quand je situe mon bras, quand je décris ma posture, quand j'essaie de préciser le lieu exact d'une douleur. Ce n'est pas tant que ce savoir est impossible à prendre en défaut; c'est plutôt qu'il s'accomplit d'une manière caractéristique, en l'absence de tout repère spatial et de tout calcul ou inférence. Un tel "savoir" non inférentiel sous-tend tout autre savoir d'orientation. Seul celui qui éprouve la différence de la droite et de la gauche, du haut et du bas, à partir de sa propre spatialité corporelle, peut aussi s'orienter dans l'espace environnant; seul, il peut situer des objets à sa droite ou à sa gauche, et ainsi de suite. "Ma" droite et "ma" gauche ne sont pas connues de la même manière que le sont la "droite" et la "gauche" en tant que propriétés des choses, c'est-à-dire en référence à mon corps. Il faut pouvoir éprouver sa propre latéralisation pour pouvoir assigner une situation à des objets. Seule la situation de mes membres est perçue sans inférence, sans indices ni repères d'aucune sorte, d'un savoir sans recherche ni observation, car elle fournit une base pour toute assignation d'un lieu ou d'une direction, et donc pour toute orientation en général. Mes sensations et les positions de mes membres possèdent une localisation primaire qui ne fait appel à aucun référentiel mais constitue le référentiel pour toute autre localisation, pour toute localisation secondaire. Mon corps se révèle ainsi d'emblée orienté et, dans cette mesure, il est la source de toute autre orientation. Quant à mon orientation dans le monde, c'est une opération complexe qui fait appel aux deux sortes de localisation, primaire et secondaire.

Le corps phénoménal se caractérise donc par une spatialité directement éprouvée et, en ce sens-là, “immédiate." Il est cette spatialité première en tant que le lieu même de ma spatialisation. C'est pourquoi, je ne suis pas situé à côté de mon corps comme une conscience transcendantale; je suis de plain pied avec le monde par mon corps et mon corps est ma propre voluminosité primordiale, celle que je suis seul à pouvoir occuper dans la mesure où je l'expérimente d'une manière à nulle autre pareille. C'est la

Journal of French and Francophone Philosophy | Revue de la philosophie française et de langue française Vol XXI, No 2 (2013) | http://www.jffp.org | DOI 10.5195/jffp.2013.611 
raison pour laquelle le corps dont nous faisons état n'est pas la chair husserlienne: cette dernière, en tant que couche esthésique des protolocalisations tactiles, possède sa propre spatialité, mais elle n'est justement pas située dans l'espace que Husserl appelle "objectif": elle en constitue l'origine pré-spatiale. C'est pourquoi elle n'a pas vraiment de limites à l'intérieur de cet espace, et Merleau-Ponty ne sera pas mal inspiré de l'appeler un "corps glorieux."22 Mais notre corps est aussi le lieu où s'exercent des dispositions et des pouvoirs. S'éprouver comme corps c'est se mesurer à des tâches qui ne sont pas d'abord de connaissance mais d'action et dont la perception elle-même dépend en son principe. Notre corps est le lieu de notre puissance d'exister qui est d'abord puissance de nous mouvoir et d'agir. Il est donné à lui-même à travers cette praxis. On peut alors, si l'on veut, définir le corps phénoménal comme l'organe d'un "je peux," à la manière de Husserl, mais à condition de comprendre autrement que lui la manière dont ce pouvoir s'exerce.

Parce qu'il n'envisage la chair que comme un objet, certes "subjectif," mais néanmoins distinct de la conscience, Husserl ne peut penser le "je peux" corporel que comme un effet d'un "je peux" égologique, et donc la spontanéité du mouvement corporel que comme l'effet d'une action que l'ego exercerait sur sa chair. La chair est le seul objet sur lequel "je règne et gouverne" ${ }^{23}$, comme il l'écrit, elle est "mon organe total," ${ }^{24} c^{\prime}$ est-à-dire à la fois "l'organe de la volonté" et "l'organe de l'esprit." ${ }^{25}$ Comment entendre ces formules? La réponse est qu'il faut les entendre en un sens littéral, au sens où la chair demeure un organon, un instrument, pour la mise en œuvre d'une action qui se révèle être en dernière instance celle de l'ego pur. Ainsi, le corps charnel ne se meut pas, il est mis en mouvement par l'ego, et "se mouvoir" reste ici un verbe transitif: "Le sujet [...] est un ego auquel appartient une chair en tant que champ de localisation de ses sensations; il [sc. l'ego] a la "faculté" ("Vermögen") ("je peux") de mouvoir librement cette chair et par conséquent les organes en lesquels ce corps s'articule." ${ }^{26}$ Certes, Husserl s'efforce de distinguer un mouvement spontané, qui est celui du vivant en général, et le mouvement mécanique des choses; mais, lorsqu'il s'agit de décrire ce mouvement spontané, il le conçoit à nouveau comme un mouvement conféré au corps par l'ego et il conçoit le corps comme le substrat sur lequel une action s'exerce. ${ }^{27}$ Or, le "je peux" corporel ne peut être analysé de cette manière. Se mouvoir corporellement, ce n'est pas agir sur son corps comme il serait possible d'agir sur un outil et, à travers lui, sur une matière donnée. Au contraire, toute action sur quoi que ce soit mobilise déjà les puissances de mon corps, en sorte que l'idée même d'une action sur mon corps présuppose le corps comme véritable sujet d'action. Je ne peux agir sur mon corps (au sens d'une action transitive) que si je possède déjà un corps capable d'agir par lui-même (c'est-à-dire d'agir sans que j'agisse sur lui), et par conséquent, un corps avec lequel je ne fais qu'un dans mon pouvoir et mon efficace sur le monde - car, dans le cas contraire, nous aboutirions inévitablement à une régression à l'infini. En d'autres termes, 
une action spontanée n'est ni une action transitive (une action sur quelque chose), ni une action réfléchie (une action où le sujet se prendrait lui-même pour objet de son action), c'est une action intransitive (exprimée par un verbe pronominal) dans laquelle il n'y a qu'un seul sujet d'action, l'agent corporel, le sujet incarné, et rien sur quoi il agisse, surtout pas lui-même ou son propre corps. De même que tout à l'heure l'expérience corporelle de soi n'était pas l'expérience d'un objet qui se trouverait par surcroît être "soi," mais une expérience dans laquelle le sujet et l'objet étaient identiques, c'està-dire dans laquelle il n'y avait pas du tout d'objet, de même, à présent, l'action de se mouvoir n'a qu'un seul agent et aucun "patient" sur lequel elle s'exerce. C'est parce que mon corps est le sujet même de mes pouvoirs que je ne suis pas "dans" mon corps d'une manière mystérieuse, ni n'agis "avec" mon corps comme avec une espèce d'instrument (un proto-instrument, pourrait-on dire), mais que je suis mon corps agile et agissant. Le corps est le lieu même de mon agilité d'agent et non quelque chose sur quoi il me faudrait agir pour me mettre moi-même en action. C'est cela que signifie la spontanéité du "se mouvoir." Dans le "se mouvoir," le seul sujet de l'action c'est moi-même-en-tant-que-corps, et nullement un ego distinct de ce corps qui devrait exercer sur lui une mystérieuse efficace.

L'analyse de l'action corporelle spontanée manifeste ainsi à nouveau selon une autre perspective combien le corps phénoménal est éloigné de toute "chose" ou "objet" et impossible à confondre avec eux. Il est l'espace que j'occupe et le lieu de ma présence au monde, et non une "possession" ou une "dépendance" aussi intimes qu'on voudra.

\section{Expérience et corporéité}

Ces affirmations conduisent-elles à envisager différemment le problème que nous avons laissé pour l'instant de côté, celui du statut de l'autre corps ou du corps de l'autre? Ce problème est l'indice, chez Husserl, de difficultés considérables. Si la chair ne se constitue comme telle qu'à travers l'expérience de l'autocontact, comment pouvons-nous découvrir que le corps d'autrui est, lui aussi, une "chair"? Il ne suffit pas de répondre qu'il ressemble étonnamment au nôtre par ses expressions et ses mouvements; car, comme le faisait remarquer Descartes, la possibilité reste ouverte que l'autre homme se révèle être en fin de compte un automate, une imitation parfaite. Mais la même difficulté n'affecte-t-elle pas notre propre description? La sensibilité affective autocentrée à travers laquelle j'éprouve mon corps ne sépare-t-elle pas ce corps du corps d'autrui aussi sûrement que chez Husserl? N'enveloppe-t-elle pas dès lors le risque d'un solipsisme, du moment que je suis le seul à posséder un corps phénoménal?

En un sens, cette objection semble toucher juste: l'écart entre la manière dont je suis conscient de mon propre corps et celle dont je suis conscient du corps d'autrui ne saurait être plus grand; car je ne peux pas ressentir la faim ou la soif, la douleur ou la fatigue de quelqu'un d'autre. Mais la

Journal of French and Francophone Philosophy | Revue de la philosophie française et de langue française

Vol XXI, No 2 (2013) | http://www.jffp.org | DOI 10.5195/jffp.2013.611 
conséquence, pour autant, ne suit pas: la conception que nous avançons est si loin d'être solipsiste, même en puissance, qu'elle fournit au contraire des arguments contre la tendance inexorablement solipsiste de la philosophie moderne depuis Descartes. Mais pour l'apercevoir, il est nécessaire d'élargir quelque peu notre perspective et de considérer notre conception dans son cadre d'ensemble. Il faut revenir à ce que nous disions en commençant et montrer pourquoi il est nécessaire de rompre de manière bien plus résolue que ne l'a fait Husserl avec les prémisses sous-jacentes à l'image moderne de la nature, telle qu'elle sous-tend l'opposition même entre naturalisme et idéalisme, pour reformuler à nouveaux frais le problème du corps phénoménal.

D'après la "nouvelle gnose" héritée de la révolution galiléenne, l'esprit demeure étranger à la nature dans laquelle prend place notre corps, il forme un second royaume qui ou bien dérive causalement du monde objectif et, dans cette mesure, peut faire l'objet d'une réduction à des processus neurophysiologiques sous-jacents, ou bien lui demeure totalement irréductible. Dans les deux cas, l'esprit est le siège de nos expériences conscientes qui, dans cette mesure, peuvent être qualifiées de "subjectives" - par contraste avec les réalités de la nature objective. Il n'y a rien de commun entre nos expériences conscientes subjectives et la réalité objective dont elles sont les expériences. Mais dire que notre expérience du monde elle-même est "subjective" en ce sens radical, c'est-à-dire qu'elle est une caractéristique de notre esprit, ou encore, faire de cette expérience un pur "état mental" (réductible ou non à un état neuronal) ne peut conduire qu'à rendre incompréhensible l'apparition même d'autrui dans le monde. Comment, sur ce théâtre intérieur de la conscience, sur cette scène de part en part privée, car appartenant à ma conscience, pourraient bien apparaître d'autres subjectivités, c'est-à-dire des subjectivités distinctes de moi, et même irréductibles à moi, des subjectivités qui ne sont pas moi? Un tel cadre de pensée ne peut dès lors donner lieu qu'à deux affirmations contradictoires et à une oscillation interminable de l'une à l'autre: 1) autrui apparaît dans mon monde, dans le monde de mon expérience, mais seulement à titre d'objet et non à titre de sujet; 2) autrui doit apparaître à titre de sujet, mais alors il ne peut que transcender absolument mon monde. Aucune de ces deux affirmations n'est satisfaisante, car le seul "fait" dont puisse partir une description phénoménologique d'autrui est sa présence dans le monde à mes côtés. Ne faut-il pas, dès lors, aller jusqu'à interroger cette prémisse implicite? Est-il vrai, en effet, que le monde de l'expérience soit mon monde? Est-il vrai que l'expérience que je fais de ce monde soit mon expérience?

Il y a plusieurs façons d'entendre ces affirmations, et partant plusieurs manières de concevoir cette "subjectivité" de l'expérience. Nous nous limiterons ici à trois: 1) L'expérience (et le monde dont cette expérience est l'expérience) sont "subjectifs" au sens où ils sont relatifs à ma perspective ou à mon point de vue; 2) L'expérience (et le monde dont cette expérience est

Journal of French and Francophone Philosophy | Revue de la philosophie française et de langue française Vol XXI, No 2 (2013) | http://www.jffp.org | DOI 10.5195/jffp.2013.611 
l'expérience) sont "subjectifs" au sens où ils dépendent causalement de mon corps; ils sont donc génériquement subjectifs; 3) L'expérience (et le monde dont cette expérience est l'expérience) sont "subjectifs" au sens où ils sont des constituants de mon esprit ou de ma conscience (laquelle se réduit, ou non, à des processus neurophysiologiques): il sont "en moi," c'est-à-dire "dans mon esprit," ils sont donc intrinsèquement subjectifs, subjectifs "dans leur étoffe même." Entre ces trois sens s'introduit une espèce de gradation.

Il est manifeste que le troisième sens rend pratiquement désespérée toute tentative pour conférer à l'autre corps, à autrui "en chair en en os," la moindre possibilité d'apparaître dans mon monde, c'est-à-dire en fin de compte dans ma conscience. Si le monde est "mien" ou "subjectif" en ce sens-là, comment une autre subjectivité, une subjectivité totalement irréductible à moi-même, pourrait-elle y apparaître? Comment un autre que moi pourrait-il être présent "en moi," dans ma conscience, être fait de l'étoffe de mes pensées et de mes vécus (Erlebnisse), tout en demeurant distinct de moi? L'idée d'une expérience intrinsèquement subjective rend inconcevable la présence même d'autrui au sein de cette expérience ou le "partage" de cette expérience avec autrui, et tous les expédients conceptuels que l'on pourra introduire à partir de là (l'Einfühlung, la constitution d'autrui par "transfert analogique," etc.) viendront trop tard pour modifier cette situation. Cette aporie, qui fut celle de la cinquième Méditation cartésienne de Husserl, ne peut être dépassée aussi longtemps que le dispositif qui sous-tend toute cette problématique n'est pas questionné dans son principe.

Le second sens devrait à première vue se ramener au troisième. Si nous concédons, en effet, que notre corps est la source causale de notre perception et de notre expérience du monde en général, est-ce que nous ne concédons pas du même coup que l'expérience est intrinsèquement subjective? En vérité, cette conséquence ne suit pas, pour une simple et bonne raison: c'est que le corps dont nous parlons ici, le corps conçu comme matrice causale de l'expérience, n'est justement pas le corps phénoménal. Tandis qu'il appartient par essence à notre corps phénoménal de se révéler à nous, dans l'expérience que nous en faisons, comme le lieu de notre présence dans le monde et au monde, et, par voie de conséquence, comme identique à nous-même, comme "subjectif" de part en part (si l'on veut adopter ce lexique de la "subjectivité"), il n'en va absolument pas de même de notre corps physiologique en tant que matrice causale de notre perception. Quelle que soit la manière dont il faille décrire ce corps, quels que soient les dispositifs physiques et physiologiques qui sous-tendent notre expérience des choses et les processus causaux qui se déroulent à ce niveau à notre insu, il n'y a aucune raison de considérer que ce corps-là soit "nôtre" en quelque sens que ce soit: c'est un dispositif physique que nous vivons d'ignorer et auquel ne s'appliquent pas les distinctions du "subjectif" et de l' "objectif," du "mien" et de 1 ' "étranger à moi-même," lesquelles ne s'appliquent au contraire qu'au

Journal of French and Francophone Philosophy | Revue de la philosophie française et de langue française Vol XXI, No 2 (2013) | http://www.jffp.org | DOI 10.5195/jffp.2013.611 
monde de notre expérience. En d'autres termes - et par là nous revenons à notre point de départ -, ce corps-objet n'est rien d'autre qu'une construction théorique parfaitement légitime au niveau qui est le sien, mais tout à fait déplacée dans une description du monde de la vie. S'il n'y a aucun sens dans lequel nous puissions dire à bon droit que ce corps-objet, en tant que matrice causale, est "subjectif," il n'y a non plus aucune raison d'identifier le second et le troisième sens de la "subjectivité" de l'expérience. On peut parfaitement affirmer que l'expérience possède sa matrice causale dans un corps physique et physiologique (celui qui peut m'être éventuellement attribué au moyen d'une inférence), sans avoir pour cela à soutenir qu'elle serait subjective dans son étoffe même. Mais alors, en quel sens peut-on affirmer d'elle qu'elle est "mon" expérience?

Ce sens est le sens le plus faible, le plus "minimal," c'est-à-dire le premier sens. Mon expérience est mon expérience dans la mesure où elle est relative à ma perspective sur le monde; et le monde sur lequel ouvre cette expérience est mon monde dans la mesure où il dépend dans sa manière même d'apparaître du point de vue que je possède sur lui. Bien sûr, ce point de vue est tel qu'il peut en principe être occupé par n'importe qui. Lorsque je ne verrai plus la rue depuis cette fenêtre, autrui pourra la voir d'ici exactement comme je la vois en ce moment. Cependant, c'est un trait phénoménologique essentiel de toute perception que mon corps phénoménal y apparaisse toujours au centre, logé pour ainsi dire en son cœur et mettant le monde en perspective à partir du point de vue qu'il occupe. L'expérience est le mode même d'apparaître des choses pour moi un "moi" corporel occupant une position toujours centrale, un "ici central ultime" comme dirait Husserl. Il s'agit là d'une des caractéristiques les plus fondamentales du corps phénoménal, une caractéristique qu'il convient à nouveau de prémunir contre un certain nombre de malentendus possibles.

En effet, dire que j'occupe une centralité absolue par mon corps dans le champ même de mon expérience consciente, ce n'est pas dire que mon corps serait une espèce d'objet - un objet "mien" sans être tout à fait moi — qui prendrait place, à son tour, dans un champ transcendantal intrinsèquement subjectif, puisque appartenant à ma subjectivité constituante et relevant de sa clôture égologique absolue. Au contraire, pour l'ensemble des raisons que nous avons détaillées, ce corps est l'espace de présence que j'occupe au milieu des choses, et le champ dans lequel il prend place n'est "subjectif" ou "mien" qu'en un sens beaucoup plus faible, au sens où — justement — je suis logé en son centre. Affirmer que mon champ d'expérience est "subjectif" et dire que j'en occupe corporellement le centre reviennent rigoureusement au même: la première affirmation se ramène à la seconde. Ainsi, mon expérience n'est ni un "état mental" ni un ensemble de vécus intrinsèquement subjectifs: elle est uniquement la manière même dont le monde se révèle à moi — un monde étranger à moi-même et qui n'est pas fait de mon étoffe - avec tout ce qui lui appartient, à commencer par des

Journal of French and Francophone Philosophy | Revue de la philosophie française et de langue française Vol XXI, No 2 (2013) | http://www.jffp.org | DOI 10.5195/jffp.2013.611 
subjectivités corporelles étrangères. L'expérience est relative à la position que j'occupe en elle par mon corps et à la manière dont mes propres mouvements en modifient le déroulement, mais elle n'est pas "subjective" au sens fort, c'est-à-dire subjective "dans son étoffe même." Ce qui est frappant, du point de vue phénoménologique, est plutôt que l'expérience ne possède aucune étoffe et ne fait aucunement écran entre moi et l'apparaître des choses (et des autres): elle est cet apparaître lui-même. Ainsi, bien loin que la reconnaissance d'une certaine "mienneté" de l'expérience, au premier sens (purement perspectif) du terme, entraîne la nécessité d'adopter le troisième sens, celui qui a constitué la toile de fond de toute la tradition cartésienne, elle-même issue de la révolution scientifique moderne, jusqu'à Husserl et même au-delà de lui, cette reconnaissance conduit au contraire à écarter cette possibilité comme source de difficultés sans fin. La vérité est que l'expérience (et le monde sur lequel elle ouvre) ne sont "miens" qu'en un sens faible, et c'est pourquoi ils sont susceptibles d'accueillir autrui dans sa présence corporelle sans que cette affirmation ne soulève la moindre difficulté. Ce que Heidegger a tenté de ressaisir grâce à son concept de Mitsein, sans pourtant aller jusqu'au bout de la révolution de pensée que ce concept appelle, est en vérité le seul point de départ d'une phénoménologie de l'autre, et d'une phénoménologie tout court.

C'est pourquoi l'expérience de notre propre corps au travers de ce que nous avons appelé une "sensibilité affective," quoique entièrement distincte de l'expérience que nous pouvons faire du corps d'autrui, ne dresse entre nous aucune barrière infranchissable, ne nous condamne à aucun solipsisme. Pas plus que celle du corps des autres et des choses en général, elle ne prend place sur aucun "théâtre intérieur." Or, c'est la supposition d'un tel théâtre, et elle seule, qui a pour conséquence inévitable le solipsisme. Notre expérience $n$ 'est pas un état mental, une scène privée sur laquelle le monde se "produirait" (ou se reproduirait, c'est-à-dire s'annoncerait par procuration), une interface entre les choses et nous; elle est le mode d'apparaître des choses et du monde. Loin d'être un champ transcendantal, et par conséquent subjectif dans sa nature même, le champ phénoménal dans lequel je figure en tant que corps est neutre à l'égard de la distinction moi / autrui - non, bien sûr, au sens où nous pourrions échanger nos places, car il appartient essentiellement à ce champ de se dévoiler à partir de 1'"ici central" que j'occupe, et que je suis le seul à occuper, mais au sens où nous pouvons tous deux y apparaître, dans une indéfectible parité et où il est le site de notre rencontre. Mon corps n'est que ma perspective sur un monde partagé avec autrui $a b$ initio et dont le partage est un trait phénoménologique originaire.

Il s'ensuit qu'autrui n'a nul besoin, pour apparaître, de transcender le monde. C'est dans le monde, dans le champ phénoménal qui est le "mien" au sens (minimal) où je l'occupe centralement avec mon corps, qu'il fait son entrée, ou qu'il a déjà fait son entrée depuis toujours. Sa présence est

Journal of French and Francophone Philosophy | Revue de la philosophie française et de langue française

Vol XXI, No 2 (2013) | http://www.jffp.org | DOI 10.5195/jffp.2013.611 
d'entrée de jeu corporelle. Et bien sûr, je n'ai pas à attribuer à autrui un corps de chair sur la base de l'apparition d'un corps-objet, car un tel corps-objet n'apparaît jamais et que son corps est d'emblée celui d'un vivant. Autrui ne se déclare pas à moi comme une conscience "logée" dans un corps ou comme un au-delà du monde y faisant irruption de manière incompréhensible, comme un infini trouant le fini ou une transcendance demeurant éternellement réfractaire à mon immanence. Il se présente comme identique à son corps de telle sorte que ce corps est sa présence même dans le monde et nullement un indice de cette présence. Identique à son corps, sans que cette affirmation n'entraîne aucun naturalisme. Le "corps" dont nous parlons, en effet, reste un corps apparaissant et non un substrat neurophysiologique auquel il s'agirait de "réduire" la conscience. Et ce corps, s'il est (d') autrui, n'épuise pas les descriptions que je puis donner de lui. Autrui, c'est assurément aussi un ensemble de capacités et de dispositions qui outrepassent sa simple "donnée" corporelle, des projets, des désirs, des aspirations sans nombre, une conscience de moi-même et du monde. Mais cette conscience, à son tour, n'a pas besoin d'être réifiée ni conçue comme une instance indépendante. Il faut dire d'autrui ce que je peux dire de moimême: avoir conscience, ce n'est pas être une conscience. Ce n'est pas parce que "conscience" est un substantif qu'il faudrait concevoir la conscience comme une espèce de chose. Plutôt que de postuler "une" conscience dans laquelle le monde devrait prendre place, une scène intérieure privée, il serait préférable d'employer "avoir conscience" comme un syntagme verbal. Ce qui existe, ce n'est pas "la" conscience, c'est plutôt un sujet corporel doué $\mathrm{d}^{\prime}$ aptitudes diverses et, parmi elles, celle d'avoir conscience du monde qui l'entoure et des autres qui y prennent place.

Nous apercevons à quel point tout est lié: le rejet du mythe de la description unique et de sa présupposition centrale selon laquelle le monde physique constituerait $1^{\prime \prime \prime}$ en soi" des choses, en tant que ce mythe sous-tend l'opposition entre le transcendantalisme et le naturalisme; la réévaluation du monde de la vie dans lequel prend place le corps phénoménal; la nécessité de distinguer bien plus nettement que ne l'a fait Husserl ce corps phénoménal du corps-objet en tant qu'idéalisation scientifique; l'expérience de ce corps comme "mien" au moyen d'une sensibilité affective qui doit être décrite comme une expérience intégrale impossible à ramener à la seule "réversibilité" du touchant-touché, et portant sur mon corps, non comme un "objet" distinct de moi dans lequel je me localiserais à titre secondaire, mais comme le volume que j'occupe au milieu des choses et la perspective sur le monde qu'il découpe; la différence radicale entre mon expérience de mon corps et celle du corps d'autrui et, par voie de conséquence, la différence de statut phénoménologique entre nos deux corps phénoménaux, mais aussi, et dans le même temps, le fait qu'ils apparaissent l'un et l'autre à l'intérieur d'une même trame apparaissante pouvant les accueillir tous deux dans leur diversité et leur hétérogénéité - à l'intérieur d'une seule et même expérience qui ne peut être conçue, dès lors, comme intrinsèquement

Journal of French and Francophone Philosophy | Revue de la philosophie française et de langue française Vol XXI, No 2 (2013) | http://www.jffp.org | DOI 10.5195/jffp.2013.611 
subjective, et donc comme un ensemble d'états mentaux ou d'Erlebnisse. Penser le corps en phénoménologie, loin d'être une entreprise simple et immédiate, exige en réalité de repenser de l'histoire de la philosophie dans son ensemble, d'interroger cette histoire de la manière la plus radicale qui soit et de remettre en cause certaines de ses thèses les plus centrales. C'est pourquoi le problème du corps n'est pas seulement un des problèmes, unes de tâches de la phénoménologie. Il l'oblige à se repenser elle-même de part en part.

${ }^{1}$ Charles Baudelaire, “Que diras-tu ce soir, pauvre âme solitaire,” Les Fleurs du mal, XLII, dans CEuvres complètes (Paris: Gallimard, Bibliothèque de la Pléiade, 1975), 43.

2 Pascal, Pensées, L. 201/ Br. 206.

${ }^{3}$ Leibniz, Monadologie, § 83, dans Philosophischen Schriften (Gerhardt, Hildesheim, New York: Georg Olms Verlag, 1978, VI), 621.

${ }^{4}$ Bacon, Novum Organum, trad. fr. de M. Malherbe et J.-M. Pousseur (Paris: PUF, 2001), 87.

${ }^{5}$ R. Spaemann, "Téléologie de la nature et action humaine," trad. de P. Destrée et J. Dewitte dans Etudes phénoménologiques, n²3-24, 1996, 63.

${ }^{6}$ Il faudrait ajouter qu'en prétendant ramener la nature à une infinité de systèmes matériels en interaction causale qui seraient indépendants de toute signification humaine, la réduction naturaliste (dont l'idéalisme accepte le point de départ) ignore le fait que l'idée même de “causalité" fait déjà appel à l'intervention humaine, comme l'a montré Georg-Henrik von Wright dans son œuvre maîtresse, Expanation and Understanding, puisque c'est seulement en intervenant de l'extérieur dans un système causal pour modifier l'antécédent et vérifier si le conséquent s'ensuit ou pas, qu'il devient possible de faire la différence entre ce qui est relation causale véritable et ce qui n'est que concomitance fortuite. L'idée de causalité dépend logiquement de celle d'action humaine finalisée.

7 Husserl, Ideen... II, Hua IV, 82, trad. fr. E. Escoubas, Recherches phénoménologiques pour la constitution (Paris: PUF, 1982), 123.

${ }^{8}$ Husserl, Ideen... I, Hua III 1, 113, trad. fr. P. Ricœur, Idées directrices pour une phénoménologie (Paris: Gallimard, 1950), 175.

${ }^{9}$ Husserl, Hua III 1, 82, trad. citée, 129. 
${ }^{10}$ Une précision, ici, s'impose: cette affirmation de l'antériorité du monde de la vie n'exclut absolument pas que notre perception ordinaire des choses ne soit en fait tributaire de concepts issus de notre culture, y compris évidemment de notre culture scientifique. Elle porte sur un point de droit et non sur un point de fait, car rien ne s'oppose en fait à ce que nous puissions percevoir l'orbite du soleil, par exemple, à condition de posséder le concept d'orbite. En fait, notre monde de la vie d'européens du début du XXle siècle est à la fois structuré par des concepts préscientifiques, comme ceux de soleil et de mouvement, et par des concepts scientifiques, comme ceux d'orbite et de gravité; mais il ne s'ensuit pas pour autant que le monde de la vie en général doive être structuré par de tels concepts. En droit, le monde de la vie est antérieur à ces concepts, car nous n'avons aucun besoin du concept d'orbite, par exemple, tout aussi peu que de celui de mouvement, pour pouvoir percevoir le trajet du soleil. L'antériorité de droit du monde de la vie vis-à-vis de nos productions culturelles et scientifiques n'exclut pas sa perméabilité de fait à ces productions.

${ }^{11}$ Husserl, Ideen... II, Hua IV, 153, trad. citée, 217.

12 Husserl, Ideen... III, Hua V, 124, trad. fr. D. Tiffeneau, La phénoménologie et les fondements des sciences (Paris: PUF, 1993), 150.

13 Husserl, Hua IV, 145; trad. citée, 207.

${ }^{14}$ Husserl, Hua IV, 144; trad. citée, 206. Par là, Husserl se rattache à une lignée qui remonte au moins à Fichte, sinon à Descartes. On pourra se reporter sur ce point aux Conférences sur la destination du savant, où Fichte formule sa question fondamentale sous la forme suivante: “Qu'est-ce qui autorise l'homme à appeler une partie déterminée du monde des corps son corps? Comment en vient-il à considérer cette partie comme son corps en tant qu'appartenant à son Moi, alors qu'il est pourtant directement opposé à celui-ci?" (Conférences sur la destination du savant, trad. fr. J.-L. Vieillard-Baron, Paris: Vrin, 1980, 45).

${ }^{15}$ Husserl, Hua IV, 212, trad. citée, 295.

${ }^{16}$ Husserl, Hua, XIV, 453.

17 Husserl, Hua IV, 214, trad. citée, 298. Il faut rappeler aussi le texte du § 54 des Ideen...I: “Il est certain qu'on peut penser une conscience sans chair (leibloses) et, aussi paradoxal que cela paraisse, sans âme [...]" (Hua III 1, 119, trad. citée modifiée, 182). Cependant, Husserl reconnaîtra dans la Krisis que "la façon dont nous sommes auprès de tous les objets qui sont pour nous est toujours [nécessairement?] une façon charnelle" (Hua VI, 110, trad. fr. de G. Granel, La crise 
des sciences européennes et la phénoménologie transcendantale, Paris: Gallimard, 1976, 122).

${ }^{18}$ Merleau-Ponty, Phénoménologie de la perception (Paris: Gallimard, “Tel,” 1976), 210.

${ }^{19}$ Husserl, Hua IV, 150, trad. citée modifiée, 214.

${ }^{20}$ Husserl, Hua IV, 151, trad. citée modifiée, 214.

${ }^{21}$ Merleau-Ponty, Phénoménologie de la perception, 116-117.

22 Merleau-Ponty, Le Visible et l'invisible (Paris: Gallimard, “Tel,” 1979, 195).

23 Husserl, Hua I, 128, trad. fr. M. de Launay, Méditations cartésiennes et les conférences de Paris (Paris: PUF, “Epiméthée,” 1994), 146.

${ }^{24}$ Husserl, Manuscrit D 12 IV, trad. fr. J.-F. Lavigne, dans E. Husserl, La terre ne se meut pas (Paris: Minuit, 1989), 92.

${ }^{25}$ Husserl, Hua IV, 96, trad. citée, 144.

${ }^{26}$ Husserl, Hua IV, 152, trad. citée modifiée, 215-216.

27 Il n'y a guère à s'étonner si Husserl retrouve en fin de compte une position qui n'est pas tellement éloignée de celle de Descartes et un "cogito de mouvement" qui rappelle irrésistiblement celui de son prédécesseur. On sait qu'à l'objection de Gassendi selon laquelle je pourrais inférer mon existence à partir de n'importe laquelle de mes actions, Descartes répond qu'il convient de distinguer la marche en tant qu'action dans le monde mobilisant le corps et sa machinerie, et la marche comme pure pensée de marcher qui seule possède une "certitude métaphysique (certitudo metaphysica)" telle qu'elle puisse entraîner la certitude de mon existence: ego ambulo, ergo sum n'est pas une inférence légitime, “sinon en tant que la connaissance intérieure que j'en ai est une pensée, de laquelle seule cette conclusion est certaine, non du mouvement du corps, lequel parfois peut être faux, comme dans nos songes, quoiqu'il nous semble alors que nous nous promenons, de façon que de ce que je pense me promener je puis fort bien inférer l'existence de mon esprit, qui a cette pensée, mais non celle de mon corps, lequel se promène" (Réponses aux cinquièmes objections, AT VII, 500; nous citons d'après la traduction d'Alquié). En somme, il y a bien un cogito ambulatoire, mais celui-ci ne "mobilise" que la seule pensée de marcher, et nullement la déambulation en tant que marche effective. Car tandis que les mouvements mondains de mon corps sont fort douteux, la seule certitude dont je dispose se limite à ma marche en pensée qui pourrait demeurer ce qu'elle est quand bien même il n'y aurait aucun corps ni aucun monde. Husserl ne dit pas autre chose, et son cogito ambulatoire n'est pas moins dualiste dans son principe même: "La thèse de l'être [...] peut être fausse, la chose n'existe

Journal of French and Francophone Philosophy | Revue de la philosophie française et de langue française Vol XXI, No 2 (2013) | http://www.jffp.org | DOI 10.5195/jffp.2013.611 
pas - c'est ce que je dis alors, ou quelqu'un d'autre que moi, dans le jugement critique après coup: en effet, je ne frappe rien, je ne danse pas, je ne saute pas. Mais l'évidence (le vécu évident) du "je pâtis" ou “j'agis," du “je bouge," n'en est pas pour autant affectée ni supprimée. (On peut dire que le "je frappe," “je danse," etc., est lui aussi un cogito, mais un cogito tel qu'il renferme en lui une thèse de transcendance et, même sous cette forme mixte, il recèle en lui l'"ego sum")" (Hua IV, 218, trad. citée, 303). Ce cogito mixte n'a pas un statut radicalement différent du cogito ambulatoire de Descartes qui, lui aussi, pour peu qu'il soit correctement entendu, contient l'ego sum à titre de certitude portant uniquement sur mon esprit. La suspension de la thèse du monde me laisse en présence d'un "agir" qui ne requiert aucun mouvement effectif et d'une efficace qui pourrait s'exercer même en l'absence de tout corps efficient et de tout déplacement mondain. En somme, le "je peux" charnel demeure un "je peux" en pensée qui n'a besoin pour s'exercer d'aucun pouvoir réel d'un corps réellement existant. Un tel "pouvoir" (Vermögen) demeure l'index ironique de la radicale impuissance transcendantale de l'ego pur. 